\section{Rita Villena Sarmiento ${ }^{1}$ Flor Pachas Barrionuevo ${ }^{1}$ Yhedina Sánchez Huamán ${ }^{1}$ Milagros Carrasco Loyola ${ }^{1}$}

'Docente del Departamento Académico de Odontología Social. Facultad de Estomatología. Universidad Peruana Cayetano Heredia.

\section{Correspondencia}

Rita Villena Sarmiento Honorio Delgado 430 - Lima 31, Perú. Teléfono: 381-1950, Anexo 226 e-mail: rsvillena@yahoo.com

\title{
Prevalencia de caries de infancia temprana en niños menores de 6 años de edad, residentes en poblados urbano marginales de Lima Norte
}

Villena-Sarmiento R, Pachas-Barrionuevo F, Sánchez-Huamán Y, Carrasco-Loyola M. Prevalencia de caries de infancia temprana en niños menores de 6 años de edad, residentes en poblados urbano marginales de Lima Norte. Rev Estomatol Herediana. 2011; 21(2):79-86.

\section{RESUMEN}

La prevalencia de caries de infancia temprana es un problema de salud pública prevenible y que afecta a un gran número de niños. El propósito de este trabajo fue determinar la prevalencia y severidad de la caries dental en niños de 6-71 meses de edad de comunidades urbano marginales de Lima. Se evaluaron a 332 niños con los criterios de caries dental de la OMS, con equipo no invasivo, bajo luz natural, y con técnica de rodilla-rodilla para los más pequeños. Fueron calibrados 3 odontólogos en el diagnóstico de caries dental (kappa interexaminador 0,79-0,92 y kappa intraexaminador 0,81-0,93). La prevalencia de caries dental fue de 62,3\% (IC 57,09$67,51)$, y se incrementó con la edad 10,5\% (0-11 meses), 27,3 \% (12-23 meses), 60,0\% (24-35 meses), 65,5\% (36-47 meses), 73,4\% (48-59 meses) y 86,9\% (60-71 meses). El índice ceod promedio fue 2,97 (DS 3,48), el componente cariado represento el 99,9\% del índice. Las piezas más afectadas en el maxilar superior fueron los incisivos centrales y primeras molares, mientras en el maxilar inferior fue la primera y segunda molar. Las manchas blancas activas tuvieron mayor presencia entre los primeros años de vida. Se concluye que existe alta carga de enfermedad y aumenta conforme se incrementan los meses de vida, siendo necesario plantear modelos de intervención temprana con especialistas del área.

Palabras clave: CARIES DE APARICION TEMPRANA / INFANCIA / EPIDEMIOLOGIA / SALUD PUBLICA.

Prevalence of early childhood caries in children under 6 years old, living in marginal communities in the north of Lima

ABSTRACT

Recibido : 25 de marzo de 2011

Early childhood caries is a preventable public health problem and affects a large number of children. The purpose of this study was to determine the prevalence and severity of dental caries in children 6-71 months of poor urban communities of Lima. Three hundred thirty two children were evaluated with the criteria of WHO's dental caries, with non-invasive equipment, natural light, with knee-knee technique for children. A calibration of dental caries was carried out with participation of 3 dentists (kappa interexaminer kappa 0.79-0.92 and 0.81 to 0.93 Intra). The results showed a prevalence of dental caries of $62.3 \%$ (CI 57.09 to 67.51 ), with an age dependent increase: $10.5 \%$ ( $0-11$ months), $27.3 \%$ (12 to 23 months), $60.0 \%$ (24-35 months), $65.5 \%$ (36-47 months), $73.4 \%$ (48-59 months) and $86.9 \%$ (60-71 months). The mean deft index was 2.97 (SD 3.48), the decayed component represented 99.9\% of the index. The parts most affected in the maxilla were the central incisors and first molars, while in the lower jaw was the first and second molar. Active white spots were most prevalent during the first years of life. We conclude that there is a high burden of disease and increased as they get older being necessary to create models of early intervention with specialists in the area.

Key words: EARLY CHIDHOOD CARIES / INFANCY / EPIDEMIOLOGY / PUBLIC HEALTH.

\section{Introducción}

En salud publica bucal, la caries dental constituye un desafío por ser una enfermedad crónica y prevenible. Es una enfermedad que no distingue edad, sexo, nivel social o cultural. En las primeras etapas de vida del ser humano, los dientes generalmente están libres de la enfermedad, pero a lo largo de su vida pueden adquirirla por diversos factores de orden biológico, estilos de vida, factor social y medio ambiental. La caries dental es el primer motivo de atención entre las enfermedades bucales para la Organización Mundial de la Salud (1).

Para la población infantil, la Asociación Dental Americana (ADA), el Centro de Prevención y Control de Enfermedades de los EEUU (CDC) y la Asociación de Odontopediatría (AAPD) definen a la caries de infancia temprana o Early Childhood Caries - ECC (en inglés) como cualquier tipo de lesión de caries presente en niños menores de 6 años y a la caries severa en la infancia o S-ECC cuando la lesión de caries se inicia en los incisivos deciduos superiores (2).

La prevalencia de la caries de infancia temprana es un tema muy discutido, y está relacionada a características sociales, culturales y económicas de las poblaciones (3). Entre los factores de riesgo asociados se citan al hábito de biberón, la placa bacteriana o biofilm dental, los hábitos de higiene bucal, la temprana adquisición y colonización de Streptococos mutans, el flujo y ca- 
lidad de la saliva del niño, el patrón de erupción de los dientes, las alteraciones estructurales de los tejidos duros, la presencia de flúor en el medio oral, tipo de ingesta, discapacidad física y/o mental, nivel socioeconómico, nivel educativo, desconocimiento sobre cuidados en higiene oral de padres o personal a cargo del niño, entre otros (4-6).

La falta de atención oportuna en niños ocasiona una serie de secuelas como son infecciones, problemas estéticos, dificultades para la alimentación, alteraciones del lenguaje y aparición de maloclusiones y hábitos orales, además de repercusiones médicas, emocionales y financieras. Se le ha considerado como un factor de riesgo para el desarrollo de caries futura en la dentición primaria y permanente, asi como su asociación con anomalías cardiacas congénitas y endocarditis bacteriana (7).

La magnitud de la caries de infancia temprana es diversa en los reportes científicos. Walter et al. (3) en una revisión de la literatura sobre caries dental citan al grupo liderado por Toverud quienes en los años 50 reportaron una prevalencia de $5 \%, 10 \%$, $50 \%$ y $75 \%$ en niños americanos de 1, 2, 3 y 5 años de edad respectivamente. Agregan que Morita et al. en 1993 identificaron en menores de 12 meses de edad una prevalencia de $3,8 \%$, entre los 13 a 24 meses $24,3 \%$ y entre 25 a 36 meses el 45,9\%.

Chu et al. en Hong Kong (8) (1999) reportaron el perfil de caries dental en niños entre los 4 a 6 años de edad. El ceod promedio global fue de 1,6 (DS 2,9). La experiencia de caries dental fue de 0,$9 ; 1,8$ y 3,3 a las edades de 4, 5 y 6 años de edad respectivamente. Con respecto al patrón de caries dental los dientes anteriores superiores tuvieron ma- yor experiencia de caries (ceod $=0,6$ ) en comparación a los antero inferiores (ceod $=0,1)$. Los dientes posteriores inferiores tuvieron más alta experiencia de caries (ceod $=0,5$ ) que los dientes posteriores superiores (ceod $=0,4)$, no encontrándose diferencia por sexo.

Mora y Martínez (9) (2000) en un estudio en niños entre los 2 a 5 años de edad, encontraron una prevalencia de caries de $37 \%$, siendo las prevalencias a las edades de 2 , 3 , 4 y 5 años de $14 \%, 41,7 \%, 43,8 \%$ y $52,5 \%$ respectivamente, con diferencia estadísticamente significativa entre ellas. El ceod promedio fue de 1,76 de dientes afectados. Respecto a la distribución de piezas afectada, el primer molar inferior derecho ocupo el primer lugar, seguido de los molares superiores. Los únicos dientes no afectados fueron los caninos inferiores.

Leite y Ribeiro (10) (2000) en Brasil en un estudio en niños de 2 a 6 años de edad encontraron que el $49,4 \%$ tenía caries dental y el ceod promedio fue de 2,03, sin diferencia por sexo. A los 2, 3, 4, 5 y 6 años de edad el porcentaje de niños con caries dental fue de 23,8\%, 35,3\%, $54,3 \%, 56,0 \%$ y $60,0 \%$ respectivamente. Respecto al número de piezas cariadas según edades referidas, los valores fueron 0,$7 ; 1,4 ; 1,9 ; 2,4$; y 2,9 respectivamente.

Kruger et al. (11) (2005) en niños pre escolares de 2 a 5 años de edad de una zona rural de Australia encontraron un $60 \%$ de prevalencia de caries dental, con un ceod promedio de 2,95 (SD 3,7), siendo 3,65 (SD 4,20) y 2,25 (SD 3,09) los promedios ceod para niños y niñas respectivamente. Hallaron que la prevalencia de caries de infancia temprana severa fue de $34 \%$ y en este grupo el promedio de ceod fue de $6,16(\mathrm{SD} 3,85)$ y para los que no te- nían caries severa el promedio fue 1,28 (SD 2,32), con diferencia significativa.

Vargas y Ronzio (12) (2006) señalan que en Estados Unidos a través del estudio nacional de nutrición y salud, NHANES III-1988-1994, el $23,7 \%$ de los niños entre los 2 a 5 años de edad han tenido experiencia de caries dental y $18,7 \%$ no ha recibido ningún tratamiento. Al evaluar el número de dientes cariados y obturados se encontró que el promedio para niños de familias de bajos ingresos fue 1,49 y en los de familias con mayores recursos 0,31 ; según grupos étnicos se evidencia disparidad, siendo que los niños blancos no hispánicos tuvieron un promedio de 0,67 piezas cariadas u obturadas, los negros no hispánicos 1,04 y los americanos mexicanos de 1,71 .

Bravo et al. (13) (2007) en una evaluación de pre escolares de España hallaron a la edad de 3 años, una prevalencia de caries dental del $17,4 \%$ aumentando a los 4 años a $26,2 \%$. Los valores ceod a los 3 y 4 años de edad fueron 0,52 y 0,76 respectivamente y el ceos fue $0,89 \mathrm{y}$ 1,35 para las mismas edades. Según la distribución de la caries dental por tipo de piezas a los 3 años de edad, las segundas molares inferior fueron las más afectadas $(6,8 \%)$ y del sector anterior el incisivo central superior $(4,1 \%)$, similar patrón se observo en la arcada inferior.

Lyny, Assdi y Sgan-Glenr (14) (2007) en Jerusalén reportaron en niños de 12 a 36 meses de edad una prevalencia de caries de infancia temprana de $17,6 \%$. Los niños tuvieron más alta prevalencia que las niñas pero sin diferencia significativa. El ceod promedio fue de 0,57 ; pero entre los niños con la enfermedad el promedio fue de 3,2.

Declerk et al. (15) (2008) encon- 
traron en Bélgica que el 6,9\% de los pre escolares tenía experiencia de caries dental a los 3 años de edad y $30,8 \%$ a los 5 años de edad. A los 3 años de edad, el 6,1\% de niños tuvo un ceod entre 1 a 4 , mientras a los 5 años el 23,6\% tuvo un ceod entre estos mismos valores.

Wyne (16) (2008) en Arabia Saudita reportó un $74,8 \%$ de prevalencia de caries dental en niños entre 3 a 5 años de edad. El ceod promedio fue de 6,10 (DS 3,9), con un componente cariado de 4,66; componente perdido de 0,54 y componente obturado de 0,92 ; no encontrándose diferencia significativa según genero. Al evaluar la prevalencia y severidad de la caries dental por tipo de escuela, los pertenecientes al gobierno tuvieron mayores valores que los privados. Los dientes posteriores más afectados fueron la segunda molar mandibular $(54,3 \%)$ y entre los dientes anteriores, el incisivo central maxilar (27,9\%). La mayoría de niños (50,3\%) tuvieron dientes afectados tanto anterior y posteriormente, $42,2 \%$ solo los dientes posteriores y muy pocos niños $(7,5 \%)$ solo el sector anterior.

En Perú, Sedano (17) (1987) encontró una prevalencia de caries dental de 94,5\% en niños de zona urbano marginales de Canto Grande. El índice ceod y ceos tuvieron valores promedios de 6,41 y 10,85 respectivamente, encontrando diferencia significativa sólo a la edad de 3 años donde los niños tienen mayor prevalencia de caries dental que las niñas. Los dientes deciduos más frecuentemente afectados por caries fueron las molares y los incisivos superiores; la superficie dentaria más frecuentemente afectada por caries fue la superficie oclusal seguida de las superficies proximales y finalmente las superficies vestibular y lingual.

Magallanes (18) (1992) en un estudio de caries dental en niños en edad pre-escolar de 3 a 5 años de edad, de 4 distritos de Lima encontró una prevalencia de $84,4 \%$, siendo inversa la relación entre prevalencia y el grado de desarrollo urbano. Asimismo que la prevalencia se incremento con la edad, pero sin diferencia significativa.

Campodonico (19) (1996) encontró una prevalencia de caries dental de $14 \%$ en niños de 6 a 42 meses de edad, que acudían a Consulta Pediátrica Externa del Policlínico Santa Rosa del distrito de Comas y del Hospital Nacional Cayetano Heredia del distrito de San Martín de Porras.

Flores (20) (1996) señala una prevalencia de caries dental (caries de biberón) de 8,8\% luego de evaluar a niños entre los 6 y 42 meses de edad que acudieron a establecimientos de salud.

Tello (21) (2001) reportó una prevalencia de caries dental de 19,1\% en niños entre los 6 a 12 meses, del $59,4 \%$ entre los 13 a 24 meses de edad y del 79,3\% entre los 25 a 36 meses de vida, que acudieron con sus madres para atenderse en una clínica de bebes en Lima.

Melgar (22) (2001) evaluó la prevalencia de caries dental en niños de 6 a 36 meses de edad del Comité de Salud Túpac Amaru del distrito de Independencia en Lima y encontró que la prevalencia de caries dental fue de $31,5 \%$, con un ceod promedio de 1,3 y ceos de 1,5.

Bernabé et al. (23) (2006) en un reporte de caries de infancia temprana, hallaron una prevalencia de $24,2 \%$ en niños de 6 a 36 meses de edad, con edad promedio de 23,77 meses. La experiencia de caries en unidad diente fue de 1,10 (DS 2,51) y por unidad superficie fue de 1,50 (DS 3,71), no se encontró diferencia significativa por sexo. El componente cariado representó casi todo el índice con un valor medio de 1,06, seguido del componente extraído de 0,03 (DS 0,25), el componente obturado no se observo. De las veinte piezas evaluadas, las piezas más afectadas fueron las molares inferiores $(30,0 \%)$ y superiores $(15,0 \%)$ en boca.

La poca información de la magnitud y caracterización de la caries de infancia temprana en el Perú sugieren la necesidad de realizar estudios epidemiológicos válidos y desarrollar programas de intervención en etapas temprana de vida para plantear acciones de promoción y prevención de primer nivel de atención.

El propósito de este estudio fue determinar la prevalencia y severidad de la caries dental en niños menores de 71 meses de edad de comunidades urbano marginales de Lima.

\section{Material y métodos}

El presente estudio de tipo descriptivo y transversal evaluó las condiciones de caries dental en niños de 0 a 71 meses de edad, pertenecientes a 7 comunidades urbano marginales del cono norte de Carabayllo, departamento de Lima, que asistieron a control de niño sano en un establecimiento del Ministerio de Salud (MINSA). El cálculo del tamaño muestral se efectúo con la formula $n=z^{2} p(1-p) / E^{2}$, sin conocimiento del tamaño de la población. El nivel de confianza (z) tuvo el valor 1,96; la proporción (p) fue igual al valor 0,24 tomado de una población similar (23). El error absoluto con valor 0,05. La muestra estimada fue de 280 niños, pero se evaluaron 332 niños.

Se incluyeron niños de familias 
del ámbito jurisdiccional del establecimiento de salud y con residencia de al menos un año, sin presencia de alteraciones sistémicas. Se utilizaron los parámetros de la OMS para la detección de caries dental (24), empleando luz natural y equipo no invasivo. Además se registro presencia y ausencia de mancha blanca. La técnica de rodilla-rodilla fue utilizada durante la evaluación clínica. Un proceso de calibración de caries dental se desarrolló con participación de tres odontólogos (valor kappa interexaminador 0,79-0,92 y kappa intraexaminador 0,81-0,93). La información se ingreso a una base de datos en programa Excel y se procesó luego en SPSS versión 10. Se analizaron las variables involucradas: lesión de caries, tipo de pieza afectada, presencia de mancha blanca activa o lesión inicial de caries no cavitada, y las covariables edad y sexo. Se determinó proporciones, medias, desviación estándar y medianas. Se evalúo con un nivel de confianza del 95\%.

Tabla 1. Prevalencia de caries dental según grupos de edad, en niños de 0-71 meses de edad, Carabayllo, 2007.

\begin{tabular}{|c|c|c|c|c|c|}
\hline edad (meses) & $n$ & \multicolumn{2}{|c|}{ Pzas anter** Pzas poster** } & Total* & IC $95 \%$ \\
\hline $0-11$ & 19 & 10,5 & 0,0 & 10,5 & $7,21-13,79$ \\
\hline $12-23$ & 44 & 9,1 & 22,6 & 27,3 & $22,51-32,09$ \\
\hline $24-35$ & 45 & 20,0 & 57,8 & 60,0 & $54,73-65,27$ \\
\hline $36-47$ & 84 & 25,0 & 60,7 & 65,5 & $60,39-70,61$ \\
\hline $48-59$ & 79 & 38,0 & 72,7 & 73,4 & 68,65-78,15 \\
\hline $60-71$ & 61 & 32,8 & 86,2 & 86,9 & $83,28-90,53$ \\
\hline$<$ de 71 & 332 & 25,9 & 64,4 & 62,3 & $57,09-67,51$ \\
\hline
\end{tabular}

La Tabla 2, muestra un ceod promedio de 2,97 (DS 3,48), con un valor mínimo y máximo de 0 y 16 respectivamente y con una mediana de 2.

La experiencia de caries de infancia temprana fue 0,42 (DS 1,61) entre los 0-11 meses de edad, de 0,50 (DS 1) de 12-23 meses de edad, de 2,18 (DS 2,81) entre los 24-35 meses de edad, de 2,80 (DS 3,08) entre los 36-47 meses de edad, de 4,78 (DS 4,28) entre los 48-59 meses de edad y de 4,03 (DS 3,19) entre los 60-71 meses de edad.

El promedio del componente cariado fue de 2,69 (DS 3,48) representando el 99,9\% del índice, mientras el componente extraído por caries fue de 0,003 (DS 0,05) representando el $0,05 \%$. No se encontró valor en el componente obturado.

En el grupo de niños con historia de caries (ceod $>0$ ), se observó que los valores promedios para el ceod fueron más elevados ( $x=4,77$ DS 3,30 ) que el obtenido para toda la muestra, en todos los grupos de edad. No se halló diferencias significativas por sexo en la experiencia de caries $(p>0,05)$.

Se identificó que conforme se incrementaba la edad, el porcentaje de niños libres de caries dental disminuyó, siendo el valor más alto (89,5\%) en 60-71 meses y el más bajo $(13,1 \%)$ en $0-11$ meses de edad (Fig.1).

Para la evaluación del tipo de pieza dental afectada por caries dental

Tabla 2: ceod y componentes según grupos de edad, en niños de 0-71 meses de edad, Carabayllo, 2007.

\begin{tabular}{llllllll}
$\begin{array}{l}\text { Edad } \\
\text { (meses) }\end{array}$ & $\begin{array}{l}\text { ceod } \\
\text { (DS) }\end{array}$ & Min & Max & $\begin{array}{l}\text { c (DS) } \\
\text { c/ceod }\end{array}$ & $\begin{array}{l}\text { e (DS) } \\
\text { e/ceod }\end{array}$ & $\begin{array}{l}\text { o (DS) } \\
\text { o/ceod }\end{array}$ & $\begin{array}{l}\text { ceod } \\
\text { (DS)* }\end{array}$ \\
\hline $0-11$ & $0,42(1,61)$ & 0 & 7 & $0,42(1,61) ; 100$ & $0(0) ; 0$ & $0(0) ; 0$ & $4,00(4,24)$ \\
$12-23$ & $0,50(1,00)$ & 0 & 5 & $0,50(1,00) ; 100$ & $0(0) ; 0$ & $0(0) ; 0$ & $1,83(1,11)$ \\
$24-35$ & $2,18(2,81)$ & 0 & 11 & $2,18(2,81) ; 100$ & $0(0) ; 0$ & $0(0) ; 0$ & $3,63(2,80)$ \\
$36-47$ & $2,80(3,08)$ & 0 & 14 & $2,80(3,08) ; 100$ & $0(0) ; 0$ & $0(0) ; 0$ & $4,27(2,86)$ \\
$48-59$ & $4,78(4,28)$ & 0 & 16 & $4,77(4,27) ; 99,8$ & $0,01(0,11) ; 0,17$ & $0(0) ; 0$ & $6,52(3,69)$ \\
$60-71$ & $4,03(3,19)$ & 0 & 13 & $4,03(3,19) ; 100$ & $0(0) ; 0$ & $0(0) ; 0$ & $4,64(2,98)$ \\
Total & $2,97(3,48)$ & 0 & 16 & $2,69(3,48) ; 99,9$ & $0,00(0,05) ; 0,05$ & $0(0) ; 0$ & $4,77(3,30)$ \\
\hline *excluye niños sin lesión de caries
\end{tabular}


y presencia de mancha blanca activa se agrupó a la muestra en dos subgrupos de 0-35 y de 36-71 meses de edad. Las Figs. 2 y 3 muestran el porcentaje de piezas afectadas del maxilar superior e inferior respectivamente, en niños de $0-35$ meses de edad. Las manchas blancas afectaron predominantemente a los incisivos centrales superiores y primeras molares inferiores.

El resultado de piezas afectadas en el maxilar superior e inferior en el grupo de niños de 36-71 meses de edad, se muestra en las Figs. 4 y 5. La mancha blanca activa afectó más a las segundas molares. El canino inferior no presento manchas blancas activas.

\section{Discusión}

La prevalencia de caries dental hallada en este estudio fue de $62,3 \%$, para la muestra de niños menores de 72 meses de edad, mayor a lo reportado por Leite (49,4\%), Vargas (23,7\%) y por Mora y Martínez (37\%) en niños de edades similares y de Brasil, Estados Unidos y España, respectivamente. Al comparar por edades, la prevalencia de caries encontrada es más alta que la de otros estudios. En niños de 12 a 23 meses se encontró una prevalencia de $27,3 \%$, cifra superior a lo reportado para la edad de un año por Walter et al. (5\%), Morita (3,8\%) y Tello (19,1\%). Entre los 24 a 35 meses de edad, este estudio halló una prevalencia de $60,0 \%$ similar a la de Tello (59,4\%), mientras que para dos años de edad Walter et al., Mora, Morita y Leite, la prevalencia es de $10 \%, 14 \%, 24,3 \%$ y $23,8 \%$, respectivamente. Entre los 36 a 47 meses de edad se encontró una prevalencia del 65,5\% mayor a la reportada en niños de tres años por Declerck

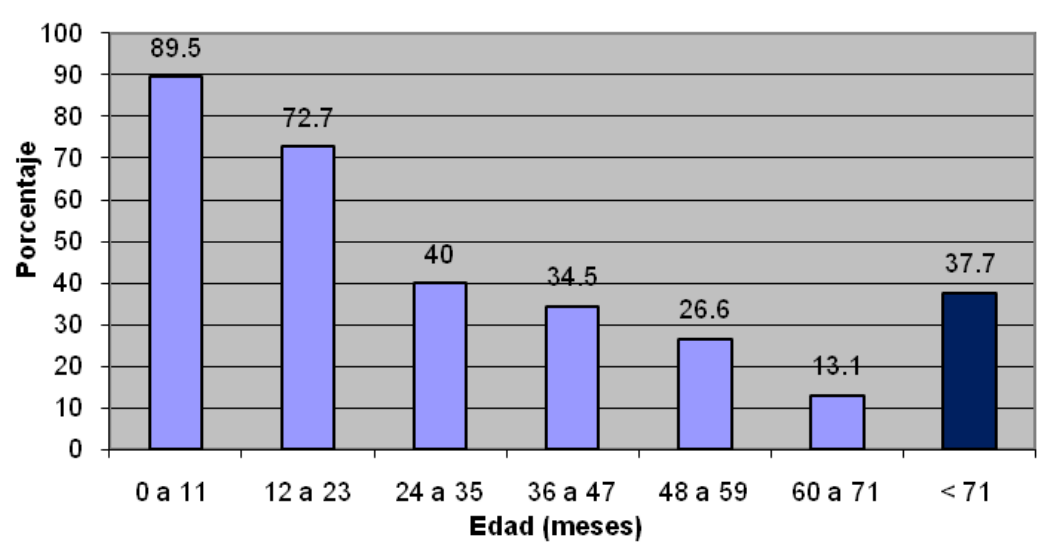

Fig. 1. Porcentaje de niños menores de 71 meses de edad libres de caries de infancia temprana. Carabayllo, 2007.

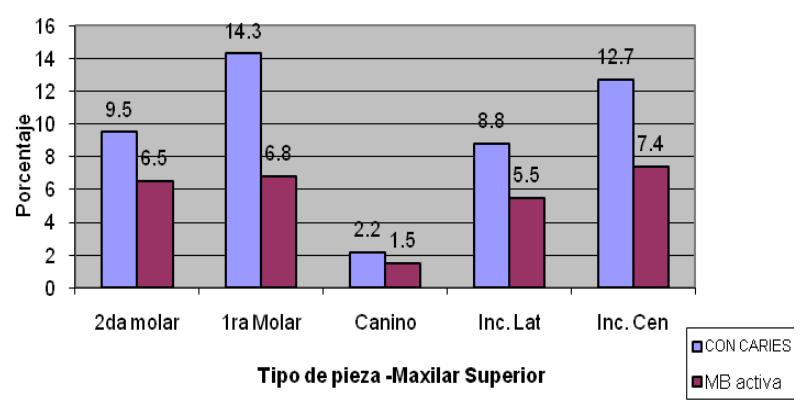

Fig. 2. Porcentaje de piezas del maxilar superior con lesiones de caries cavitadas y no cavitadas en niños de 0 a 35 meses de edad, Carabayllo, 2007. 2007. et al. (6,9\%), Bravo (26,2\%), Leite $(41,7 \%)$, Morita $(45,9 \%)$ y Walter et al. (50\%), pero siendo menor a lo reportado por Tello (79,3\%). Entre los 48 a 59 meses este estudio encontró $73,4 \%$ cifra mayor a la encontrada para la edad de cuatro años de edad por Mora y Leite (43,8\% y $54,3 \%$ respectivamente). Entre los 60 a 71 meses de edad se halló una prevalencia de $86,9 \%$ siendo mayor a lo encontrado por Walter et al. (75\%), Leite (56\%\%), Mora (52,5\%) y Declerck et al. (30,8\%).

La diferencia de la magnitud de la prevalencia de caries dental entre los niños de un año y los dos años de edad se podría explicar por la ventana de infección que ocurre generalmente durante los primeros 19 meses de vida, la erupción de todos los dientes deciduos, dificultando la buena higiene, además de cambios dietéticos, algunas prácticas inadecuadas de alimentación durante la ablactancia, dieta cariogénica, lactancia nocturna, así como el empleo de biberones y falta de higienización a sus niños, como lo reportan diversas investigaciones. (25).

Un estudio también realizado en el cono norte de Lima que evaluó el perfil de la atención odontológica de los infantes en los servicios de salud reportó un bajo porcentaje de niños con diagnòstico de caries dental, siendo atendidos a los $4(4,6 \%)$ y 5 (7,2\%) años de edad (26). Este va-

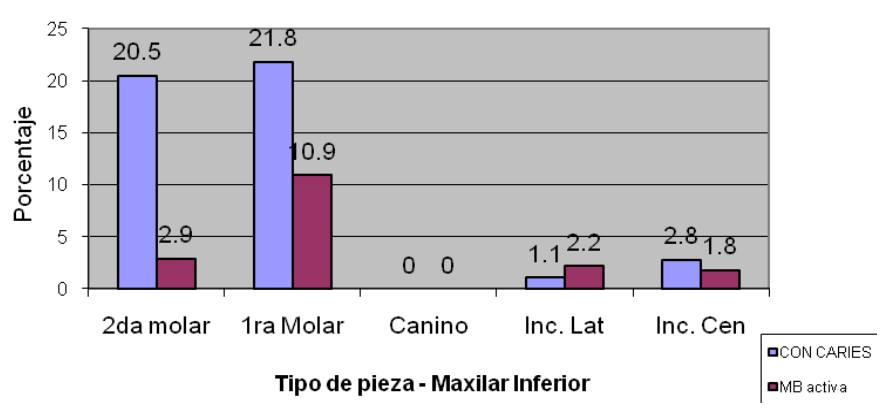

Fig. 3. Porcentaje de piezas del maxilar inferior con lesiones de caries cavitadas y no cavitadas en niños de 0 a 35 meses de edad, Carabayllo, 


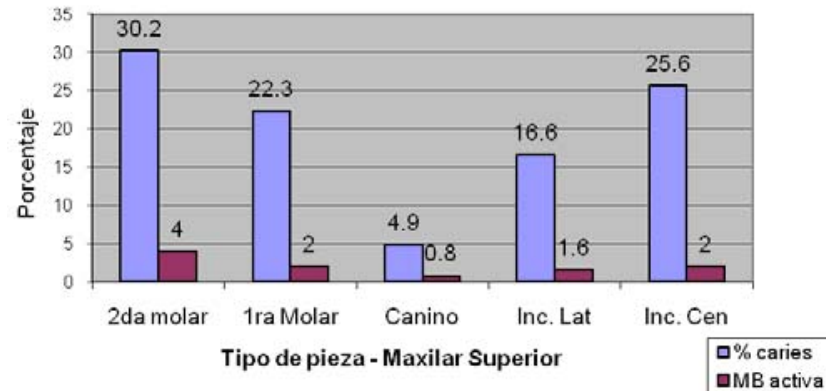

Fig. 4. Piezas con lesiones de caries cavitadas y no cavitadas en el maxilar superior de niños de 36 a 71 meses de edad, Carabayllo, 2007.

lor contrasta notoriamente con la prevalencia encontrada en el estudio. Probablemente la falta de educación de las madres y padres en reconocer lesiones de caries dental y la baja priorización a los problemas bucales frente a otras necesidades de salud, hace que no acudan de forma oportuna a los servicios de atención odontológica.

$\mathrm{Al}$ evaluar el grupo de niños comprendidos entre los 36 a 71 meses de edad, el estudio encontró una prevalencia de $74,1 \%$ siendo menor a lo reportado por Sedano (94,55\%) en un contexto urbano marginal donde prima la extrema pobreza a fines de los años ochenta y Magallanes $(84,4 \%)$ en cuatro distritos de características sociales medio bajo a inicios de los años noventa. Este menor valor se podría explicar entonces como una tendencia a la disminución de la prevalencia de caries a lo largo del tiempo, sustentada por programas como la fluoruración de la sal, los distintos contextos de donde procedían los niños, así como por factores metodológicos. Lamentablemente no contamos con valores nacionales en infancia que nos permitan sustentar estás hipótesis.

Igualmente al evaluar el grupo de niños comprendidos entre los 0 a 35 meses de edad, el estudio encontró una prevalencia de 37,9\% siendo mayor a los reportes de Livny, Assali

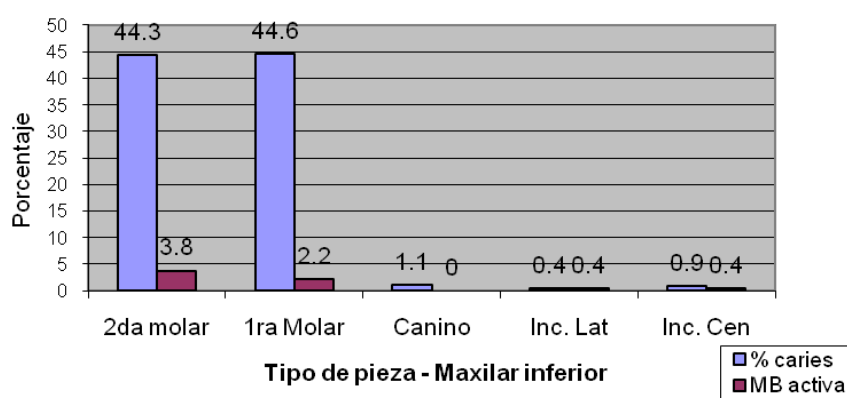

Fig. 5. Piezas con lesiones de caries cavitadas y no cavitadas en el maxilar inferior de niños de 36 a 71 meses de edad, Carabayllo, 2007.

y Sgan-Cohen $(17,6 \%)$ en Jerusalén, lo que puede explicarse a la dieta tradicional de la tribu de Beduinos de Jerusalén con escaso consumo de productos procesados. En comparación con estudios realizados en el país, la prevalencia de caries para el mismo grupo de edad es similar al de Melgar (31,5\%), posiblemente porque se realizó en comunidades de nivel socioeconómico semejantes y el levantamiento de los datos de caries fue realizado por una especialista en odontopediatría; sin embargo, difiere al estudio de Bernabé (24,2\%), el cual a pesar de haberse realizado en comunidades de características similares, el levantamiento de la información estuvo a cargo de estudiantes de pregrado.

La experiencia de caries dental en el estudio $(2,97)$ fue cercana a la reportada por Leite $(2,03)$ en un grupo de niños de 2 a 6 años de edad, mayor a la citada por Mora $(1,76)$ y Vargas $(1,49)$ en niños de 2 a 5 años de edad y tanto de Melgar $(1,3)$ y Bernabé $(1,1)$ en niños de 6 a 36 meses de edad. Representó casi la mitad de lo reportado por Sedano $(6,41)$ en un grupo de niños de 3 a 5 años de edad. Estas diferencias se explicarían por el número de evaluados, rango de edades y situación del contexto social.

Los valores de ceod por edades en comparación a otros estudios fueron superiores $(8,10,13)$.
Similarmente a los otros reportes, la mayor carga de enfermedad estuvo confinada al componente cariado, existiendo poca evidencia de acceso a servicios de salud y si lo hacen es solo por mutilación. Esto concuerda con el poco uso de los servicios odontológicos $(4,9 \%)$ en niños menores de 6 años que como fue reportado acuden a los establecimientos públicos (26).

Cabe resaltar que se halló a la primera molar inferior como la pieza más afectada por caries dental, en concordancia con otros estudios $(8,9,13,16,17,23)$, lo que se podría explicar por la anatomía de la pieza. Similarmente a lo descrito en otras investigaciones el incisivo central superior ocupó el segundo lugar de afección $(8,13,16,17)$ entre el total de piezas afectadas. Muy poco compromiso se observó en los incisivos inferiores entre un rango de edad meses a otro de mayor edad. La explicación a tal patrón de ataque se vería explicado por el posicionamiento y la protección que la lengua confiere a los incisivos inferiores. Otras de las variables evaluadas fue la lesión de mancha blanca activa, observándose que en los primeros años de vida de 0 a 35 meses de edad aparecen en mayor proporción en incisivos y molares superiores, pero su presencia desciende conforme se incrementa la edad. Estos hallazgos son próximos 
a lo reportado en niños de 5 años de edad de una población Turka pero con el empleo de criterio de diagnóstico de Nyvad. Probablemente muchas de estas lesiones iniciales se transforman a lesiones cavitadas e irreversibles por falta de un tratamiento preventivo oportuno para hacer reversible este proceso.

Se concluye que la prevalencia de caries dental es alta en infantes residentes en comunidades de bajos recursos y se incrementa con la edad $(p=0,00)$ tanto en piezas anteriores como posteriores $(p<0,05)$. La experiencia de caries, tiene un incremento significativo y evidente a partir de los 24 meses de edad ( $<<0,05)$, por lo que se evidencia una necesidad de atención temprana en odontología.

\section{Agradecimiento}

A la Facultad de Estomatología Roberto Beltrán, por apoyo financiado al Proyecto de Infantes en poblados urbano marginales $\left(\mathrm{N}^{\circ}\right.$ SIDISI 52281). Los resultados formaron parte de la etapa de diagnóstico. Al Puesto de Salud Juan Pablo II de Carabayllo, y las facilidades brindadas por el médico jefe Dr. Mario Suarez Rodríguez.

\section{Referencias bibliográficas}

1. Daly B, Watt R, Batchelor P, Treasure E. Essential Dental Public Health. New York: Oxford University Press; 2002.

2. Villena RS. Caries de infancia temprana. II Encuentro Peruano de Odontología para Bebes, Lima-Perú. Libro de resúmenes del congreso, 2006. [citado Febrero 2010]. Disponible en: http:/ /www.odontobebeperu.org/ acuerdos/02-01.pdf

3. Walter L, Ferelle A, Issao M. Odontología para el bebe. Sao Paulo: Editora Artes Medicas
Ltda; 2000.

4. Arango MC, Baena GP. Caries de la infancia temprana y factores de riesgo: revisión de la literatura. Rev Estomat. 2004; 12(1):59-65.

5. Mahejabeen R, Sudha $P$, Kulkarni SS, Anegundi R. Dental caries prevalence among preschool children of Hubli: Dharwad city. J Indian Soc Pedod Prev Dent. 2006; 24(1):19-22.

6. Selwitz RH, Ismail AI, Pitts NB. Dental caries. Lancet. 2007; 369(9555):51-9.

7. Begzati A, Berisha M, Meqa K. Early childhood caries in preschool children of Kosovo - a serious public health problem. BMC Public Health. 2010; 10:788.

8. Chu CH, Fung DS, Lo EC. Dental caries status of preschool children in Hong Kong. Br Dent J. 1999; 187(11):616-20.

9. Mora León L, Martínez Olmos J. Prevalencia de caries y factores asociados en niños de 2-5 años de los Centros de Salud Almanjáyar y Cartuja de Granada capital. Aten Primaria. 2000; 26(6):398-404.

10.Leite IC, Ribeiro RA. Dental caries in the primary dentition in public nursery school children in Juiz de Fora, Minas Gerais, Brazil. Cad Saude Publica. 2000; 16(3):717-22.

11. Kruger E, Dyson K, Tennant M. Pre-school child oral health in rural Western Australia. Aust Dent J. 2005; 50(4):258-62.

12. Vargas CM, Ronzio CR. Disparities in early childhood caries. BMC Oral Health. 2006; 6 Suppl 1:S3.

13. Bravo Peréz M, Llodra Calvo JC, Cortés Martinicorena FJ, Casals Peidro E. Encuesta de
Salud Oral de Preescolares en España 2007. RCOE. 2006; 12(3):143-68.

14.Livny A, Assali R, Sgan-Cohen HD. Early Childhood Caries among a Bedouin community residing in the eastern outskirts of Jerusalem. BMC Public Health. 2007; 7:167.

15. Declerck D, Leroy R, Martens L, Lesaffre E, Garcia-Zattera MJ, Vanden Broucke S, Debyser M, Hoppenbrouwers K. Factors associated with prevalence and severity of caries experience in preschool children. Community Dent Oral Epidemiol. 2008; 36(2):168-78.

16. Wyne AH. Caries prevalence, severity, and pattern in preschool children. J Contemp Dent Pract. 2008; 9(3):24-31.

17.Sedano L. Estudio epidemiológico de salud oral en niños de 3 a 5 años de edad en poblaciones urbano marginadas de Canto Grande, distrito de San Juan de Lurigancho, Lima Metropolitana [Tesis de Bachiller en Estomatología] Lima: UPCH, 1987.

18. Magallanes M. Estudio epidemiológico de salud oral en niños en edad pre-escolar en 4 distritos de Lima Metropolitana [Tesis de Bachiller en Estomatología] Lima: UPCH, 1992.

19. Campodónico C. Relación del uso de fluoruros y la prevalencia de caries dental en niños de 0 a 42 meses de edad atendidos en Consulta Pediátrica Externa del Policlínico Santa Rosa del distrito de Comas y del Hospital Nacional Cayetano Heredia del distrito de San Martin de Porras durante los meses de diciembre de 1995 y enero de 1996 [Tesis de Bachiller en Estomatología] Lima: UPCH, 1996. 
20. Flores J. Factores de riesgo para la caries de biberón en niños de 6 a 42 meses de edad, que acudieron a consulta pediátrica durante el mes de Diciembre de 1995 y Enero de 1996 al Policlínico Santa Rosa (Comas) y el Hospital Nacional Cayetano Heredia (SMP) [Tesis de Bachiller en Estomatología] Lima: UPCH, 1996.

21. Tello P. Estudio epidemiológico de la prevalencia de caries y su relación con hábitos alimentarios y de higiene bucal en niños de 6 a 36 meses de edad. Ceo .com.pe. [revista en Internet]. [citado Febrero 2010]. Disponible en:http:/ /www.ceo.com.pe/005_revista_ art01.htm

22. Melgar R. Prevalencia de caries de la infancia temprana según determinantes sociodemográficos, conductuales, nutricionales y relacionados a la transmisión temprana de microorganismos en un grupo de infantes del comité zonal de salud de Túpac Amaru distrito de Independencia, LimaPerú. 1998 [Tesis de Magíster en Estomatología] Lima: UPCH, 2002.

23. Bernabé E, Delgado-Angulo E, Sánchez P. Resultados de un sistema para la vigilancia de la caries de la infancia temprana. Rev Med Hered. 2006; 17(4): 227-33.

24.OMS. Encuestas de Salud Bucodental. Métodos básicos. Ginebra, 1997.

25.Carrasco M, Villena R, Pachas F, Sánchez Y. Lactancia mater- na y hábitos de succión nutritivos y no nutritivos en niños de 0 71 meses de comunidades urbano marginales del cono norte de Lima. Rev Estomatol Herediana. 2009; 19(2):83-90.

26.Pachas F, Sánchez Y, Carrasco M, Villena R. Perfil de atención de salud en gestantes y niños de 0- 71 meses de edad, de un Puesto de Salud del Cono Norte Carabayllo, Lima-Perú. Rev Estomatol Herediana. 2008; 18(2):83-92.

27.Kuvvetli SS, Cildir SK, Ergeneli S, Sandalli N. Prevalence of noncavitated and cavitated carious lesions in a group of 5year-old Turkish children in Kadikoy, Istanbul. J Dent Child (Chic). 2008; 75(2):158-63. 